\title{
Incidence of Surgical Site Infection and Factors Associated among Cesarean Deliveries in Selected Government Hospitals in Addis Ababa, Ethiopia, 2019
}

\author{
Hana Lijaemiro, Semarya Berhe Lemlem $(\mathbb{D}$, and Jembere Tesfaye Deressa \\ Addis Ababa University College of Health Sciences, Department of Midwifery, Addis Ababa, Ethiopia \\ Correspondence should be addressed to Jembere Tesfaye Deressa; jembere_tesfaye@yahoo.com
}

Received 6 November 2019; Revised 10 December 2019; Accepted 2 January 2020; Published 22 February 2020

Academic Editor: Curt W. Burger

Copyright (c) 2020 Hana Lijaemiro et al. This is an open access article distributed under the Creative Commons Attribution License, which permits unrestricted use, distribution, and reproduction in any medium, provided the original work is properly cited.

\begin{abstract}
Background. One-third to two-thirds of operated patients in low-income countries acquire surgical site infection, which is nine times higher when compared to high-resource countries. Identifying the incidence and risk factors that contribute to surgical site infection following cesarean delivery is a step ahead for preventing and reducing the problem. Nonetheless, the distribution of the problem in Addis Ababa, where the rate of cesarean delivery is relatively high compared to other parts of the country, is under investigation. Objective. The aim of this study is to assess the incidence of surgical site infection among cesarean deliveries and factors associated with it in selected governmental hospitals found in Addis Ababa, Ethiopia, in 2019. Method. A hospital-based prospective cohort study design was employed to follow 175 women, who gave birth by cesarean delivery in selected government hospitals in Addis Ababa, from March 11 to April 9, 2019. Convenience sampling method was used to select study units from the randomly selected hospitals. Descriptive statistics were run for determining the rate of cesarean delivery surgical site infection. Presence and degree of association between outcome and independent variables were computed through bivariate logistic regression analysis and factors that had $p<0.2$ significance level in the bivariate logistic regression analysis were considered in the multivariable logistic regression analysis. Result. From 166 participants who completed 30-day follow-up, 25 (15\%) of the participants developed surgical site infection. Age, gestational age, duration of operation, and $\geq 5$ vaginal examinations showed a significant association with the outcome variable with AOR $(95 \% \mathrm{CI})$ of $((\mathrm{AOR}=1.504,95 \% \mathrm{CI}:(1.170-1.933, p=0.001)))$, $((\mathrm{AOR}=0.019,95 \% \mathrm{CI}:(0.001-0.291, p=0.004))),((\mathrm{AOR}=1.108,95 \% \mathrm{CI}:(1.025-1.197, p=0.009)))$, and $((\mathrm{AOR}=13.076,95 \%$ CI: $(1.018-168.002, p=0.048)))$, respectively. Conclusion and recommendation. Surgical site infection rate is higher and certain associations lost due to small sample size. Further interventional studies with vast sample size are recommended.
\end{abstract}

\section{Introduction}

Cesarean delivery, often known as cesarean section, is an operative delivery of a fetus via maternal abdominal and uterine wall incision [1]. Cesarean delivery is indicated in cases of antepartum hemorrhage and fetal distress, and CD becomes mandatory to prevent potential maternal and neonatal morbidity and mortality [2].

Medically indicated cesarean section has a pronounced influence to prevent life-threatening conditions, such as obstetric fistula and birth asphyxia, and save maternal and child life [3]. However, CD also accompanies the risk of infection, hemorrhage, and uterine rupture and placentation problems in current and future pregnancies [3-6].

Hence, in 1985, World Health Organization (WHO) declared that the optimal threshold for cesarean section rate should be $10-15 \%$ based on the data available especially from Northern Europe, where perinatal morbidities and mortalities were low with the above rate [7]. This declaration aims at enhancing the practice of performing CD for those who need it and avoid the procedure for medically ineligible clients. Even though this declaration is debatable to date, CD rate exceeding $15-20 \%$ is not yet associated with improved perinatal conditions [8]. 
Nonetheless, recent studies revealed that the rate of cesarean delivery is rising unpredictably leading to actual, potential, and lifelong maternal and neonatal complications. Half of the cesarean section procedures exceeding the WHO maximum CD rate threshold are performed in Brazil and China [8]. This can be evidenced by a $40.5 \%$ CD rate exhibited in Latin America and the Caribbean region [9]. On the contrary, the poor perinatal maternal, neonatal, and infant outcomes in low-resource countries with less than $5 \%$ CD rate may attribute to the socioeconomic situation in the countries [10]. One of the short-term morbidities which take place after cesarean section is surgical site infection (SSI). It is a postsurgery infection in parts of the body where it is performed. The Center for Disease Control and Prevention (CDC)'s National Nosocomial Infection Surveillance System serves as a de facto standard for defining and classifying SSI. It classifies SSI as incisional and organ/ space SSI. Incisional SSI, in turn, has two divisions: superficial and deep SSI. The infection on both types of incisional SSIs is restrained to the incision site only. Superficial SSI involves skin and subcutaneous tissue of the incisional site, while deep SSI distributes to the muscle and fascia of the incisional site. If the infection spreads to body wall layers that are not manipulated during the surgical procedure, it is named as organ (space) SSI [11].

The leading healthcare-associated infection (HAI) in low-income countries is SSI affecting one-third to twothirds of operated patients, which is nine times higher when it is compared to high-resource countries [12]. A study conducted in 25 low- and middle-income African countries also witnessed most complications following surgery, and $10.2 \%$ were due to surgical site infection [13]. It is also undeniable that SSI is the second most frequently reported HAI in even high-income countries such as USA and Europe [14].

The majority of the conditions leading to HAIs including SSIs are at ease of intervention. Factors such as poor waste disposal and environmental hygiene practice reduced standard precaution implementation trend and development of local procedures and guidelines for each clinical activity is feasible to be intervened by intrainstitution and national health organization divisions $[12,14]$. Governmental involvement might be necessary in fulfilling infrastructure and essential equipment for the procedure and recruiting an adequate number of staff with appropriate knowledge and skill [12]. Host-related characteristics such as advanced maternal age, raised BMI, and coexisting maternal disease might influence CD SSI. Likewise, adverse conditions surrounding pregnancy and CD would have an effect on maternal CD SSI status.

\section{Methods and Materials}

2.1. Study Setting and Period. The study was conducted from March 11 to May 24, 2019, in Addis Ababa, Ethiopia. The city was divided into 10 subcities which has 14 governmental hospitals in 2019 according to a data from Ethiopian Ministry of Health.
2.2. Study Design. A 30-day hospital-based prospective cohort follow-up of women who gave birth by CD in the first month of the data collection period was conducted.

2.3. Source Population. The source population was drawn from women who delivered by CD in government hospitals in Addis Ababa.

2.4. Study Population. The sample population was drawn from women who delivered by CD in the first 30 days of the data collection period in the selected four governmental hospitals in Addis Ababa, Ethiopia.

2.5. Inclusion Criteria. Women who delivered by CD in the study period and were willing to participate in the study, who had a permanent address for reporting their condition, and who were mentally fit to differentiate and report their status were included in the study.

2.6. Exclusion Criteria. Women who were severely ill and died during the first phase of the study period were excluded.

2.7. Sampling Methods. Among 14 public hospitals in Addis Ababa, 4 were selected by simple random sampling method. Then, based on the number of cesarean deliveries in the hospital, a proportionate number of study units were allocated for each hospital. All CDs that took place during the first phase of data collection time were included as study unit until the allocated sample size was attained (convenience sampling method).

2.8. Sample Size Determination. Single population proportion formula was utilized to calculate the sample size and adding $10 \%$ contingency sample size became 165 , $p=$ estimation of CD rate $(11 \%$ in a study done in Hawassa Teaching and Referral Hospital [15]).

\subsection{Data Collection}

2.9.1. Data Collection Procedure. All mothers who delivered by $\mathrm{CD}$ in selected government hospitals during the study period were asked for their willingness to participate in the study. The medical record of those volunteer women was reviewed for the presence of SSI risk factors. Each mother was interviewed through telephone for the development of SSI syndromes within 30 postoperative days.

2.9.2. Data Collection Tool. The data collection tool was adapted from a study done in Zimbabwe and modified based on the contextual situation. It contains questions that assess maternal characteristics such as age, gravidity, parity, BMI, coexisting morbidities; peripartum maternal conditions such as maternal health condition, PROM, number of vaginal examinations, trial of labor before $\mathrm{CD}$; and 
procedural characteristics such as duration of operation, type of surgery, type of anesthesia, qualification of the surgeon, type and timing of antibiotics administration, preoperative skin preparation, and the likes.

2.9.3. Data Analysis. The collected data were coded, entered, checked, and cleaned by Epi-data version 3.1 and were exported to SPSS version 20, for data analysis. The proportion of CD SSI was computed by running descriptive statistics, followed by bivariate and multivariable logistic regression to determine the statistical association between independent and dependent variables. Presence and degree of association between outcome and independent variables were computed through odds ratio with $95 \%$ confidence interval (CI) and $p$ value $<0.05$.

2.9.4. Data Quality Control. Data collectors were trained on the objective of the study, the data collection procedures, and the data collection tool. The data collection tool was adapted from a study done in Zimbabwe and modified based on contextual situations. The tool was pretested in $10 \%$ of similar population in another Specialized Referral Hospital to assure the validity of it and modify accordingly.

Collected data were reviewed and checked for completeness and consistency on a daily basis.

\section{Result}

A 30-day post-CD prospective follow-up through telephone for development of syndromes of SSI was conducted from April 22 to May 24, 2019, for 175 mothers who gave birth by $\mathrm{CD}$ in four selected government hospitals in Addis Ababa from March 11 to April 19, 2019. Among 175 mothers, 166 (approximately 95\%) of them completed the 30-day telephone interview, while 11 (5 from TASH, 1 from ZMGH, and 3 form Y12MCGH) of them were lost before completing the follow-up.

3.1. Sociodemographic and Obstetrics Characteristics of Participants. The age of the participants in the study ranged from 19 to 40 years, while majority of the participants (approximately 75\%) were less than 30 years old. In addition to this, around $95 \%$ of the study participants were from urban. Most of the women involved in the study had 3 or less parity status (93.4\%); among this, Para I, Para II, and Para III account for $53.6 \%, 24.1 \%$, and $15.7 \%$ of the participants, respectively.

Minimal number of the participants, $28.9 \%$, was found to have previous $\mathrm{CD}$, suggesting most $\mathrm{CDs}$ in this study were mostly due to other obstetric emergencies. Women with prolonged PROM also account for only $15.7 \%$ of the participants as presented below (Table 1).

\subsection{Operation-Related Characteristics of Participants}

3.2.1. Indication for $C D$ and Related Comorbidities Identified.
TABLE 1: Sociodemographic and obstetric characteristics of study participants $(n=166)$ who gave birth through CD in four selected government hospitals in Addis Ababa, Ethiopia, 2019.

\begin{tabular}{lcccccc}
\hline \multirow{2}{*}{ Variables } & \multicolumn{5}{c}{ Post-CD SSI } \\
& Category & \multicolumn{3}{c}{ Yes } & No & Total (\%) \\
& & $N$ & $\%$ & $N$ & $\%$ & \\
\hline \multirow{2}{*}{ Age } & $<30$ yrs & 4 & 3.3 & 120 & 96.7 & $124(74.7 \%)$ \\
& $\geq 30$ yrs & 21 & 50 & 21 & 50 & $42(25.3 \%)$ \\
Residence & Urban & 24 & 15.2 & 134 & 84.8 & $158(95.2 \%)$ \\
History of & Rural & 1 & 12.5 & 7 & 87.5 & $8(4.8 \%)$ \\
previous CD & Yes & 13 & 27.1 & 35 & 72.9 & $48(28.9 \%)$ \\
& No & 12 & 10.2 & 106 & 89.8 & $118(71.1 \%)$ \\
Parity & Para I & 11 & 12.4 & 78 & 87.6 & $89(53.6 \%)$ \\
& Para II & 7 & 17.5 & 33 & 82.5 & $40(24 \%)$ \\
& Para III & 5 & 19.2 & 21 & 80.8 & $26(15.7 \%)$ \\
PROM & Para IV & 1 & 10 & 9 & 90 & $10(6 \%)$ \\
& Para V & 1 & 100 & 0 & 0 & $1(0.6 \%)$ \\
& No & 10 & 11.4 & 78 & 88.6 & $88(53 \%)$ \\
& rupture & & & & & \\
& $\leq 12$ hrs & 4 & 7.7 & 48 & 92.3 & $52(31.3 \%)$ \\
& $\geq 12$ hrs & 11 & 42.3 & 15 & 57.7 & $26(15.7 \%)$ \\
\hline
\end{tabular}

Majority of the participants (86\%) in the study were referred from other facilities. Among them, the most common indication for $\mathrm{CD}$ in the study period was fetal distress (40.4\%), followed by CPD (19.9\%) and arrest of labour $(13.3 \%)$, while the rest is accountable for other indications such as PIH and oligohydramnios.

Since the sample size is small $(n=166)$, adequate representatives of the comorbidities hypertension (30.7\%), DM (4.8\%), and HIV (3.6\%) were not available during the study period.

3.2.2. Preoperative Preparation. Preoperative antibiotics prophylaxis was provided for around $92 \%$ of the participants, and among them, $66.3 \%$ of the participants gained $2 \mathrm{~g}$ ampicillin as a prophylaxis while the rest utilized $1 \mathrm{~g}$ ceftriaxone for the same purpose.

Most of the health professional's handwashing (70.5\%) before $\mathrm{CD}$ was conducted utilizing plain soap and water, which was available in most setups. Skin preparation for CD of all participants was done by alcohol, while iodine was utilized in addition to alcohol in approximately $20 \%$ of the participants.

As depicted in Table 2, only 59 (35.5\%) of the study participants were not clipping of the pubic hair. Among those who clipping of their pubic hair, $78(40 \%)$ were shiny on top at their home, while the rest $28(17 \%)$ and $1(0.6 \%)$ were lacking hair at preoperative wards and on the operating table, respectively.

3.3. Operative and Postoperative Characteristics. Only 36 (21.7\%) operations were conducted electively without any medical or other emergence (Table 3).

Residents perform majority of CDs (94.6\%) followed by specialists (4.8\%) and GP (general physicians) (0.6\%) during the study period. The most prevalent type of skin incision 
TABLE 2: CD indication, related comorbidities, and preoperative characteristics of study participants $(n=166)$ in four selected government hospitals in Addis Ababa, Ethiopia, 2019.

\begin{tabular}{|c|c|c|c|c|c|c|}
\hline \multirow{3}{*}{ Variables } & \multirow{3}{*}{ Category } & \multicolumn{4}{|c|}{ Post-CD SSI } & \multirow{3}{*}{ Total $(\%)$} \\
\hline & & \multicolumn{2}{|c|}{ Yes } & \multicolumn{2}{|c|}{ No } & \\
\hline & & $N$ & $\%$ & $N$ & $\%$ & \\
\hline \multirow{2}{*}{ CPD } & Yes & 10 & 30.3 & 23 & 69.7 & $33(19.9 \%)$ \\
\hline & No & 15 & 11.3 & 118 & 88.7 & $133(80.1 \%)$ \\
\hline \multirow{2}{*}{ Fetal distress } & Yes & 8 & 11.9 & 59 & 88.1 & $67(40.4 \%)$ \\
\hline & No & 17 & 17.2 & 82 & 82.8 & $99(59.6 \%)$ \\
\hline \multirow{2}{*}{ Arrest of labour } & Yes & 3 & 13.6 & 19 & 86.4 & $22(13.3 \%)$ \\
\hline & No & 22 & 15.3 & 122 & 84.7 & $144(86.7 \%)$ \\
\hline \multirow{3}{*}{ Hypertension } & Chronic HTN & 2 & 14.3 & 12 & 85.7 & $14(8.4 \%)$ \\
\hline & PIH & 10 & 27 & 27 & 73 & $37(22.3 \%)$ \\
\hline & No HTN & 13 & 11.3 & 102 & 88.7 & $115(69.3 \%)$ \\
\hline \multirow{3}{*}{$\mathrm{DM}$} & Chronic DM & 0 & 0 & 2 & 100 & $2(1.2 \%)$ \\
\hline & Gestational DM & 1 & 16.7 & 5 & 83.3 & $6(3.6 \%)$ \\
\hline & No DM & 24 & 15.2 & 134 & 84.8 & $158(95.2 \%)$ \\
\hline \multirow{3}{*}{ HIV } & Detected before pregnancy & 0 & 0 & 3 & 100 & $3(1.8 \%)$ \\
\hline & Detected during pregnancy & 0 & 0 & 3 & 100 & $3(1.8 \%)$ \\
\hline & No HIV detected & 25 & 15.6 & 135 & 84.4 & $160(96.4 \%)$ \\
\hline \multirow{2}{*}{ Referral status } & Yes & 20 & 14 & 123 & 86 & $143(86.1 \%)$ \\
\hline & No & 5 & 21.7 & 18 & 78.3 & $23(13.9 \%)$ \\
\hline \multirow{2}{*}{ Antibiotics prophylaxis } & Yes & 23 & 15 & 130 & 85 & $153(92.2 \%)$ \\
\hline & No & 2 & 15.4 & 11 & 84.6 & $13(7.8 \%)$ \\
\hline \multirow{2}{*}{ Plain soap and water use } & Yes & 20 & 14.6 & 117 & 85.4 & $137(82.5 \%)$ \\
\hline & No & 5 & 17.2 & 24 & $82.8 \%$ & $29(17.5 \%)$ \\
\hline \multirow{2}{*}{ Antimicrobial soap and water use } & Yes & 5 & 17.2 & 24 & 82.8 & $29(17.5 \%)$ \\
\hline & No & 20 & 14.6 & 117 & 85.4 & $137(82.5 \%)$ \\
\hline \multirow{2}{*}{ Client's pubic hair shiny on top } & Yes & 16 & 15 & 91 & 85 & $107(64.5 \%)$ \\
\hline & No & 9 & 15.3 & 50 & 84.7 & $59(35.5 \%)$ \\
\hline \multirow{3}{*}{ Place pubic hair bald } & Ward & 4 & 14.3 & 24 & 85.7 & $28(16.7 \%)$ \\
\hline & Operating table & 0 & 0 & 1 & 100 & $1(0.6 \%)$ \\
\hline & Home & 12 & 15.4 & 66 & 84.6 & $78(45 \%)$ \\
\hline
\end{tabular}

TABLE 3: Operative and postoperative characteristics of study participants $(n=166)$ among CDs in four selected government hospitals in Addis Ababa, Ethiopia, 2019.

\begin{tabular}{|c|c|c|c|c|c|c|}
\hline \multirow{3}{*}{ Variables } & \multicolumn{6}{|c|}{ Post-CD SSI } \\
\hline & \multirow[t]{2}{*}{ Category } & \multicolumn{2}{|c|}{ Yes } & \multicolumn{2}{|c|}{ No } & \multirow[t]{2}{*}{ Total (\%) } \\
\hline & & $N$ & $\%$ & $N$ & $\%$ & \\
\hline \multirow{3}{*}{ Type of operation } & Elective & 1 & 2.8 & 35 & 97.2 & $36(21.7 \%)$ \\
\hline & Emergency & 24 & 18.7 & 106 & 81.5 & $130(78.3 \%)$ \\
\hline & $\mathrm{GP}$ & 0 & 0 & 1 & 100 & $1(0.6 \%)$ \\
\hline \multirow[t]{2}{*}{ CD performed by } & Resident & 23 & 14.65 & 134 & 85.4 & $157(94.6 \%)$ \\
\hline & Specialist & 2 & 25 & 6 & 75 & $8(4.8 \%)$ \\
\hline \multirow{2}{*}{ Skin incision } & Transverse & 22 & 13.7 & 139 & 86.3 & $161(97 \%)$ \\
\hline & Vertical & 3 & 60 & 2 & 40 & $5(3 \%)$ \\
\hline \multirow{2}{*}{ Skin closure } & Interrupted & 2 & 9.5 & 19 & 90.5 & $21(12.7 \%)$ \\
\hline & Continuous & 23 & 15.9 & 122 & 84.1 & $145(87.3 \%)$ \\
\hline \multirow{2}{*}{ Post-CD antibiotics } & Yes & 20 & 12.8 & 136 & 87.2 & $156(94 \%)$ \\
\hline & No & 5 & 50 & 5 & 50 & $10(6 \%)$ \\
\hline
\end{tabular}

used throughout this study was transverse incision being performed in about 161 (97\%) of the study participants. CDs were completed with a range of 4 to 70 minutes; the median time to complete the operation is 26.5 minutes. Skin closure after CD was determined by the physician's choice, which was interrupted in $21(12.7 \%)$ of the study participants.

Post-CD antibiotics were provided for 156 (94\%) of the participants. Two grams of ampicillin IV was used in $70 \%$ of 


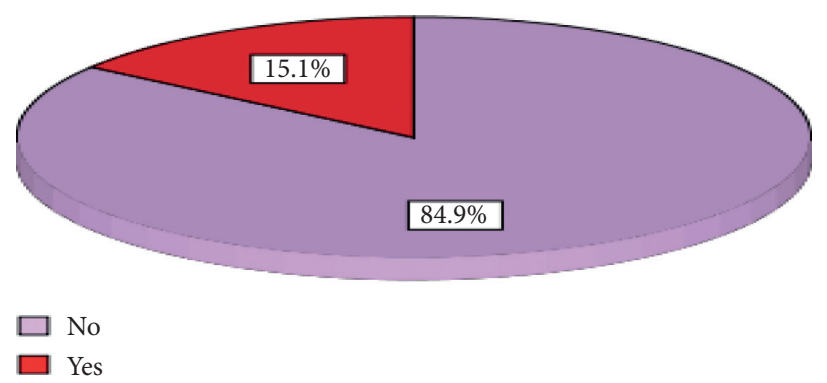

FIGURE 1: Incidence rate of SSI among women who delivered by CD in four governmental hospitals in Addis Ababa, Ethiopia, 2019.

TABLE 4: Factors associated with post-CD infection among women $(n=166)$ who gave birth by CD in four selected government hospitals in Addis Ababa, Ethiopia, from March 11 to May 24, 2019.

\begin{tabular}{|c|c|c|c|c|c|c|c|}
\hline \multirow{2}{*}{ Variable } & \multirow{2}{*}{ Category } & \multicolumn{2}{|c|}{ SSI } & \multirow{2}{*}{ COR $(95 \% \mathrm{CI})$} & \multirow{2}{*}{$p$ value } & \multirow{2}{*}{$\operatorname{AOR}(95 \% \mathrm{CI})$} & \multirow{2}{*}{$p$ value } \\
\hline & & Yes & No & & & & \\
\hline \multirow{2}{*}{ Age } & $<30$ yrs & 4 & 120 & 1 & & 1 & 0.01 \\
\hline & $\geq 30$ yrs & 21 & 21 & $1.44(1.010-2.023)$ & 0.030 & $1.5(1.170-1.933)$ & \\
\hline \multirow{3}{*}{ PROM } & No & 10 & 78 & 1 & & 1 & \\
\hline & $\leq 12 \mathrm{hrs}$ & 4 & 48 & $0.65(0.193-2.188)$ & 0.121 & $0.235(0.02-2.808)$ & 0.35 \\
\hline & $>12 \mathrm{hrs}$ & 11 & 15 & $5.72(2.064-15.85)^{*}$ & 0.142 & $3.982(0.381-41.614)$ & 0.27 \\
\hline \multirow{3}{*}{ Number of vaginal examinations } & No & 2 & 54 & 1 & & 1 & \\
\hline & $1-4$ & 5 & 36 & $2.454(0.552-10.916)$ & 0.472 & $10.732(0.438-263.006)$ & 0.34 \\
\hline & $\geq 5$ & 17 & 52 & $5.776(1.597-20.89)^{*}$ & 0.132 & $13.076(1.018-168.002)$ & 0.048 \\
\hline \multirow{2}{*}{ Length of labour trial before CD } & $\leq 24 \mathrm{hrs}$ & 10 & 108 & 1 & & 1 & \\
\hline & $>24 \mathrm{hrs}$ & 15 & 33 & $4.909(2.016-11.955)^{*}$ & 0.113 & $3.408(0.402-28.897)$ & 0.232 \\
\hline \multirow{3}{*}{ Gestational age } & $<37$ wks & 3 & 26 & $0.238(0.063-0.897)^{*}$ & 0.131 & $0.074(0.004-1.315)$ & 0.32 \\
\hline & $37-40$ wks & 5 & 80 & $0.129(0.044-0.376)^{*}$ & 0.151 & $0.019(0.001-0.291)^{* *}$ & 0.004 \\
\hline & $>40$ wks & 17 & 35 & 1 & & 1 & \\
\hline \multirow{2}{*}{ Type of operation } & Elective & 1 & 35 & 1 & & 1 & \\
\hline & Emergency & 24 & 106 & $7.925(1.034-60.734)^{*}$ & 0.145 & $7.667(0.190-309.384)$ & 0.243 \\
\hline \multirow{2}{*}{ CPD } & Yes & 10 & 23 & $3.420(1.368-8.552)^{*}$ & 0.010 & $1.860(0.158-21.857)$ & 0.120 \\
\hline & No & 15 & 118 & 1 & & 1 & \\
\hline \multirow{2}{*}{ Type of skin incision } & Transverse & 22 & 139 & 1 & & 1 & \\
\hline & Vertical & 3 & 2 & $9.477(1.498-59.964)^{*}$ & 0.010 & $0.046(0.001-2.408)$ & 0.253 \\
\hline \multirow{2}{*}{ Postoperative antibiotics } & Yes & 20 & 136 & 1 & & 1 & \\
\hline & No & 5 & 5 & $6.800(1.807-25.595)^{*}$ & 0.142 & $8.999(0.037-213.220)$ & 0.254 \\
\hline \multirow{3}{*}{ Surgical wound class } & Class I & 10 & 78 & 1 & & 1 & \\
\hline & Class II & 4 & 48 & $0.65(0.193-2.188)$ & 0.246 & $0.235(0.02-2.808)$ & 0.132 \\
\hline & Class III & 11 & 55 & $5.72(2.064-15.85)^{*}$ & 0.132 & $3.982(0.381-41.614)$ & 0.148 \\
\hline
\end{tabular}

${ }^{*} p$ value $\leq 0.2$ in bivariate analysis; ${ }^{* *} p$ value $<0.05$ in multivariable analysis.

those who received the antibiotics. Besides, all study participants did not develop PPH during the study period.

3.4. Incidence Rate of SSI. From 166 participants who completed 30-day follow-up, 25 (15\%) of the participants developed SSI depicted below (Figure 1). Among them, 17 (68\%) developed superficial SSI that only required outpatient wound dressing and use of broad-spectrum antibiotics. But $8(32 \%)$ developed deep SSI that required prolonged hospital stay.

All mothers (166) in this study were followed for 30 days starting from the first postoperative day. All post-CD SSIs were detected during the 30-day telephone interview, i.e., no
SSI was detected during the in-patient stay. Moreover, fifteen (60\%) of the SSIs were detected from days 11-17 followed by days $1-10$ which exhibits $9(36 \%)$ of SSIs and days $25-30$ that enables detection of 1 SSI.

3.4.1. Factors Associated with Cesarean Delivery among SSI. Bivariate logistic regression was run for variables in this study. Variables that scored a $p$ value of less than or equal to 0.2 were considered in multivariable logistic regression.

In the multivariate analysis, age, gestational age, and duration of operation showed a significant association with a $p$ value of $\leq 0.05$. According to the latter analysis, every one year increment in age leads to 1.5 times greater risk 
$((\mathrm{AOR}=1.504,95 \% \mathrm{CI}:(1.170-1.933)))$ to develop SSI. Similarly, every one-minute increment in the duration of operation has 1.1 times higher risk $((\mathrm{AOR}=1.108,95 \% \mathrm{CI}$ : (1.025-1.197)) for having SSI.

Giving birth by CD at term was also found to be $98.1 \%$ protective $((\mathrm{AOR}=0.019,95 \% \mathrm{CI}$ : $(0.001-0.291)))$, for the incidence of SSI compared to that of postterm deliveries. Nonetheless, association could not be made for that of preterm delivery in this study presented in Table 4.

\section{Discussion}

One hundred seventy-five women who delivered by CD in the selected hospitals in the first month (phase) of the data collection were weekly interviewed through telephone for the development of SSI syndromes for four consecutive weeks, taking the date of operation as day 1. Among them, nine (approximately 5\%) participants were lost before completing their follow-up, and therefore, they were excluded from the study.

Post-CD SSI was detected in twenty-five (15.1\%) of the participants. From this, one can appreciate the use of postdischarge infection surveillance for immediate evaluation and improvement of CD service, since post-CD inpatient stay is decreasing from time to time. The rate, however, might be underestimated due to a significant number of lost participants.

The rate, $15.1 \%$, is significantly high compared to the results of studies done in Polish hospital, US academic institution, Israel health institution, Thai-Myanmar border hospital, a hospital in Oman, two hospitals in Libya, three sub-Saharan African countries, and Tanzania which showed an incidence rate of $0.5 \%, 5.5 \%, 3.7 \%, 6.2 \%, 2.6 \%$, $2.53-3.07 \%, 7.6 \%$, and $10.9 \%$, respectively $[6,16-23]$. This difference might be due to a difference in SSI definition, distribution of risk factors among the studied group, study time, socioeconomic status, and healthcare delivery system.

Even though most of the studies reviewed in this study utilized the definition of CDC for SSI diagnosis, some, like the study in Libya, utilized more specific criteria to diagnose the issue unlike this study. For example, Libya's study utilized healing progress and the presence of certain bacteria to diagnose the condition in addition to the CDC's criteria. This might contribute to the wide difference in the incidence rate of post-CD SSI between Libya's and the present study [18].

It can also be noticed that compared to previous studies conducted in Ethiopia, the incidence rate of post-CD SSI in this study is high and this can be exemplified by the rates of $6.8 \%$ in Lemlem-Carl Hospital, $11 \%$ in Hawassa, and $11.7 \%$ in Ayder Hospital $[15,21,24]$. The difference might be due to the study design, study place, and sample size.

However, the rate is in line with a study conducted in Jordan that finds out a $14.4 \%$ incidence of post-CD SSI [25]. This might partially be due to the proximity of the study time and means of data collection used by both studies was also similar. Some of the variables considered in this study show a strong association with the outcome variable. A one-year increment in age exhibits 1.5 times greater risk
$((\mathrm{AOR}=1.504,95 \% \mathrm{CI}:(1.170-1.933)))$ to develop post-CD SSI. This result is in line with findings of studies done in Israel and Libya, which showed age increment is a risk factor for post-CD SSI $[17,18]$. This agreement might be due to reduced ability of cell growth and repair which is inevitable as age increases, and furthermore, gradual changes in endocrine and immune system with aging might also contribute.

The finding, however, contradicts that of a study performed in three sub-Saharan African countries that demonstrated younger age was associated with a higher incidence of SSI [22]. This might be due to overrepresentation of young age population and the endemic nature of the condition.

The study also showed giving birth by $\mathrm{CD}$ at term was 98.1\% protective $((\mathrm{AOR}=0.019,95 \% \mathrm{CI}:(0.001-0.291)))$ for the incidence of SSI compared to that of postterm deliveries. Even though direct association between preterm delivery and the outcome variable could not be found in this study, it agrees with studies in the USA and Israel that labouring at term can protect infection by avoiding complications of preterm labour such as hastened labour $[16,17]$.

This study revealed a one-minute increment in the duration of operation has 1.1 times higher risk $((\mathrm{AOR}=1.108,95 \% \mathrm{CI}:(1.025-1.197)))$ for having SSI. The finding was in line with a study done in Lemlem-Karl Hospital, Ethiopia [24]. This might be due to the extended time of operation that leads to more tissue damage and rise in the introduction of various microorganisms into the peritoneal cavity.

\section{Conclusion}

Rate of incidence of post-CD SSI was high in this study. The variables PROM, length of labour trial, type of operation, indication of operation, type of incision, and postoperative antibiotics were not significant in this study. However, they should be tested in a study with larger sample size which has an adequate representation of the cases.

Maternal age, gestational age, and duration of operation were found to have a strong association with the study variable.

\section{Data Availability}

All data generated or analyzed during the study were included in this published article and its additional information files. The raw data and materials are available and can be obtained from Addis Ababa University School of Nursing and Midwifery Research and Publication Committee, Ethiopia, and from the corresponding author on reasonable request.

\section{Ethical Approval}

Ethical approval was obtained from the Research Ethical Committee of Nursing and Midwifery Department, College of Health Sciences, Addis Ababa University. Written 
permission was sought from the responsible body of the study setting.

\section{Consent}

Informed consent was obtained from each participant after explained nature, purpose, and procedures of the study. Each study participant was informed about the purpose, methods of collection, anticipated benefit, and risk of study. Privacy and confidentiality of the data provided were strictly maintained. Participants were assured that their participation was voluntary and they have every right to withdraw or refuse to give information at any time in the study without compromising the service they get from the facility.

\section{Disclosure}

The funding organization has no role in the design of the study and data collection, analysis, and interpretation of data and in writing the manuscript.

\section{Conflicts of Interest}

The authors declare that they have no conflicts of interest.

\section{Authors' Contributions}

JT and HL conceptualized and designed the study. SB, HL, and JT participated in the collection of data and statistical analysis. JT, HL, and SB interpreted the data. JT and HL drafted the first manuscript. SB and JT revised it critically for intellectual content. All authors read and approved the final manuscript.

\section{Acknowledgments}

The authors would like to acknowledge the contributions of research assistants, Addis Ababa University, study participants, and selected public hospitals including management bodies during this study. Addis Ababa University funded via small grant for data collection and transport service.

\section{Supplementary Materials}

Appendix: data collection tool: Post-cesarean delivery surgical site infection data collection tool. (Supplementary Materials)

\section{References}

[1] E. K. Martin, A cost-effectiveness modelling study of strategies to prevent post-caesarian surgical site infection, Ph.D. thesis, Queensland University of Technology, Brisbane, Australia, 2017.

[2] S. M. Pfeifer, NMS Obsetetrics and Gynecology, Lippincott Williams \& Wilkins, a Wolter Kluwer Business, Philadelphia, PA, USA, 7 edition, 2012.

[3] A. P. Betran, M. R. Torloni, J. Zhang et al., "What is the optimal rate of caesarean section at population level? A systematic review of ecologic studies," Reproductive Health, vol. 12, no. 1, p. 57, 2015.
[4] J. P. Souza, A. M. Gülmezoglu, P. Lumbiganon et al., "Caesarean section without medical indications is associated with an increased risk of adverse short-term maternal outcomes: the 2004-2008 WHO global survey on maternal and perinatal health," BMC Medicine, vol. 8, no. 1, p. 71, 2010.

[5] P. Lumbiganon, M. Laopaiboon, A. M. Gülmezoglu et al., "Method of delivery and pregnancy outcomes in Asia: the WHO global survey on maternal and perinatal health $2007-$ 08," The Lancet, vol. 375, no. 9713, pp. 490-499, 2010.

[6] A. Różańska, A. Jarynowski, K. K. Godlewska et al., "Does surgical site infection after caesarean section in Polish hospitals reflect high-quality patient care or poor postdischarge surveillance? Results from a 3-year multicenter study," American Journal of Infection Control, vol. 46, no. 1, pp. 20-25, 2018.

[7] A. Betran, M. Torloni, J. Zhang, and A. Gülmezoglu, "WHO statement on caesarean section rates," BJOG: An International Journal of Obstetrics \& Gynaecology, vol. 123, no. 5, pp. 667-670, 2016.

[8] L. Gibbons, J. Belizan, J. A. Lauer, A. P. Betran, M. Merialdi, and F. Althabe, "Inequities in the use of cesarean section deliveries in the world," American Journal of Obstetrics and Gynecology, vol. 206, no. 4, pp. 331. e1-331. e19, 2012.

[9] A. P. Betrán, J. Ye, A.-B. Moller, J. Zhang, A. Metin Gülmezoglu, and M. Regina Torloni, "The increasing trend in caesarean section rates: global, regional and national estimates: 1990-2014," PLoS One, vol. 11, no. 2, Article ID e0148343, 2016.

[10] J. M. Belizán, N. Minckas, E. M. McClure et al., “An approach to identify a minimum and rational proportion of caesarean sections in resource-poor settings: a global network study," The Lancet Global Health, vol. 6, no. 8, pp. e894-e901, 2018.

[11] A. J. Mangram, "Guideline for prevention of surgical site infection centers for disease control and prevention (CDC) hospital infection control practices advisory committee," American Journal of Infection Control, vol. 27, no. 2, pp. 97-132, 1999.

[12] World Health Organisation, Health Care-Associated Infections Fact Sheet, World Health Organisation, Geneva, Switzerland, 2016.

[13] B. M. Biccard, T. E. Madiba, H. L. Kluyts, D. M Munlemvo et al., "Perioperative patient outcomes in the African surgical outcomes study: a 7-day prospective observational cohort study," The Lancet, vol. 391, Article ID 10130, 2018.

[14] World Health Organisation, Global Guidelines for the Prevention of Surgical Site Infection, World Health Organisation, Geneva, Switzerland, 2016.

[15] S. Wodajo, M. Belayneh, and S. Gebremedhin, "Magnitude and factors associated with post-cesarean surgical site infection at Hawassa university teaching and referral hospital, southern Ethiopia: a cross-sectional study," Ethiopian Journal of Health Sciences, vol. 27, no. 3, pp. 283-290, 2017.

[16] L. J. Moulton, J. L. Munoz, M. Lachiewicz, X. Liu, and O. Goje, "Surgical site infection after cesarean delivery: incidence and risk factors at a US academic institution," The Journal of Maternal-Fetal \& Neonatal Medicine, vol. 31, no. 14, pp. 1873-1880, 2018.

[17] Y. Krieger, A. Walfisch, and E. Sheiner, "Surgical site infection following cesarean deliveries: trends and risk factors," The Journal of Maternal-Fetal \& Neonatal Medicine, vol. 30, no. 1, pp. 8-12, 2016.

[18] M. M. Osela, "Study on post caesarean section wound infection at misurata central hospital and Al-khoms teaching 
hospital, Libya," Journal of Dental and Medical Sciences, vol. 15, 2016.

[19] S. Assawapalanggool, N. Kasatpibal, S. Sirichotiyakul, R. Arora, and W. Suntornlimsiri, "Risk factors for cesarean surgical site infections at a Thai-Myanmar border hospital," American Journal of Infection Control, vol. 44, no. 9, pp. 990-995, 2016.

[20] H. Dhar, I. A. Busaidi, B. Rathi, E. A. Nimre, V. Sachdeva, and I. Hamdi, "A study of post-caesarean section wound infections in a regional referral hospital, Oman," Sultan Qaboos University Medical Journal, vol. 14, no. 2, pp. e211-7, 2014.

[21] T. A. Wendmagegn, G. B. Abera, W. T. Tsehaye, K. B. Gebresslasie, and B. G. Tella, "Magnitude and determinants of surgical site infecion among women underwent cesarean section in ayder comprehensive specialized hospital Mekelle City, Tigray region, Northern Ethiopia, 2016," BMC Pregnancy Childbirth, vol. 18, no. 1, p. 489, 2018.

[22] K. Chu, R. Maine, and M. Trelles, "Cesarean section surgical site infections in sub-saharan africa: a multi-country study from medecins sans frontieres," World Journal of Surgery, vol. 39, no. 2, pp. 350-355, 2015.

[23] F. J. Mpogoro, S. E. Mshana, M. M. Mirambo, B. R. Kidenya, B. Gumodoka, and C. Imirzalioglu, "Incidence and predictors of surgical site infections following caesarean sections at Bugando Medical Centre, Mwanza, Tanzania," Antimicrobial Resistance and Infection Control, vol. 3, no. 1, p. 25, 2014.

[24] K. A. Gelaw, A. M. Aweke, F. H. Astawesegn, B. W. Demissie, and L. B. Zeleke, "Surgical site infection and its associated factors following cesarean section: a cross sectional study from a public hospital in Ethiopia," Patient Safety in Surgery, vol. 11, no. 1, p. 18, 2017.

[25] M. H. A. Jalil, K. A. Hammour, M. Alsous et al., "Surgical site infections following caesarean operations at a Jordanian teaching hospital: frequency and implicated factors," Scientific Reports, vol. 7, no. 1, p. 12210, 2017. 